\title{
New Protocol for Auditory Brainstem Implant Positioning
}

\author{
Sheila Veronese*, Marco Cambiaghi, Andrea Sbarbati
}

Department of Neuroscience, Biomedicine and Movement Sciences - Verona University, Verona, Italy

*Corresponding Author: Sheila Veronese, Department of Neurosciences, Biomedicine and Movement Sciences, Verona University - 10 Sq. A.Stefani, Verona 37134, Italy

Received date: August 20, 2021 ; Accepted date: October 29, 2021; Published date: November 12,2021

Citation: Sheila Veronese, Marco Cambiaghi, Andrea Sbarbati (2021) New Protocol for Auditory Brainstem Implant Positioning. J. Neuroscience and Neurological Surgery. 9(5); DOI:10.31579/2578-8868/203

Copyright: (C) 2021 Sheila Veronese, This is an open-access article distributed under the terms of The Creative Commons Attribution License, which permits unrestricted use, distribution, and reproduction in any medium, provided the original author and source are credited

\begin{abstract}
Background: Surgery for applying the auditory brainstem implant is an otoneurosurgery that requires careful intraoperative monitoring to optimize the placement of the electrode paddle. This study aimed to validate a new method capable of increasing the accuracy of electrode array placement, reducing channel interaction, electrical artefacts, and saturation effects, and providing the largest number of electrodes that can be activated with the lowest possible electric charge.
\end{abstract}

Materials and methods: Thirty-six subjects aged between 1.42 and 69.92 years were tested during surgery for auditory brainstem implantation. We recorded auditory electrical responses of the brainstem using the implant supplier's suggested stimulation protocol and the new protocol.

Results: Saturations effects and electric artefacts were noticed respectively in $81.85 \%$ and $53.25 \%$ of recordings using implant supplier's method, while in $70.34 \%$ and $24.75 \%$ of recordings using the new method, with a percentage variation of $11.51 \%$ and $28.50 \%$. Considering the amount of charge required to activate the electrodes, with the implant supplier's method an average charge of $14 \mathrm{nC}$ was needed, while with the new protocol an average charge of $8 \mathrm{nC}$ was necessary.

Conclusions: The new method improves the coupling between the auditory brainstem implant and the surface of the cochlear nucleus.

Keywords: ABI; EABR; intraoperative monitoring; electrodes positioning

\section{Introduction}

The auditory brainstem implant (ABI) represents a device that effectively restores auditory sensations in patients with NF2 and acoustic neuromas. Its application was extended to children and adults with injuries or malformations of the cochlea and cochlear nerve, who are not eligible for a cochlear implant [1], [2].

The use of electrophysiology, in particular of the electrical auditory brainstem responses (EABR), is known to facilitate electrode plate placement during ABI implant surgery [3], [4], [5].

Waring optimized pacing and recording parameters to achieve efficient EABR monitoring during surgery [6], [7]. Achieving efficient monitoring means being able to recognize the waveforms of auditory responses and distinguish them from the current of stimulation and other evoked potentials that could result from stimulation of nearby non-auditory anatomical structures.
Many authors have tried to define the optimum EABR monitoring procedure and to correlate its results with the auditory outcomes [8], [9], [10], [11], [12], [13].

Nevison et al. [8] demonstrated that achieving good intraoperative EABRs is not related to the performance of implanted patients, but subjects who achieve good EABRs also have postoperative auditory sensations. Furthermore, they reported the presence of non-auditory sensations during ABI programming in $92.3 \%$ of cases. This means that the number of usable electrodes varies significantly between subjects, and that an imperfect coupling of the electrodes with the cochlear nuclei can more easily induce the excitation of non-auditory stimulations.

This study aimed to validate a new method capable of increasing the accuracy of electrode array placement, reducing channel interaction, electrical artefacts, and saturation effects, and providing the largest number of electrodes that can be activated with the lowest possible electric charge. 


\section{Materials and Methods}

Surgical and electrophysiological procedures were approved by the Ethics Committee of Verona Hospital. For these procedures, an informed written consent was acquired from the patients and children's parents. This study was carried out in accordance with the Declaration of Helsinki.

Thirty-six ABI subjects aged between 1.42 and 69.92 years were tested during ABI implantation surgery. They presented different aetiologies (Figure 1). The chosen implant was the Cochlear Nucleus ABI24M (Cochlear Ltd., Sydney, Australia), for all the patients.

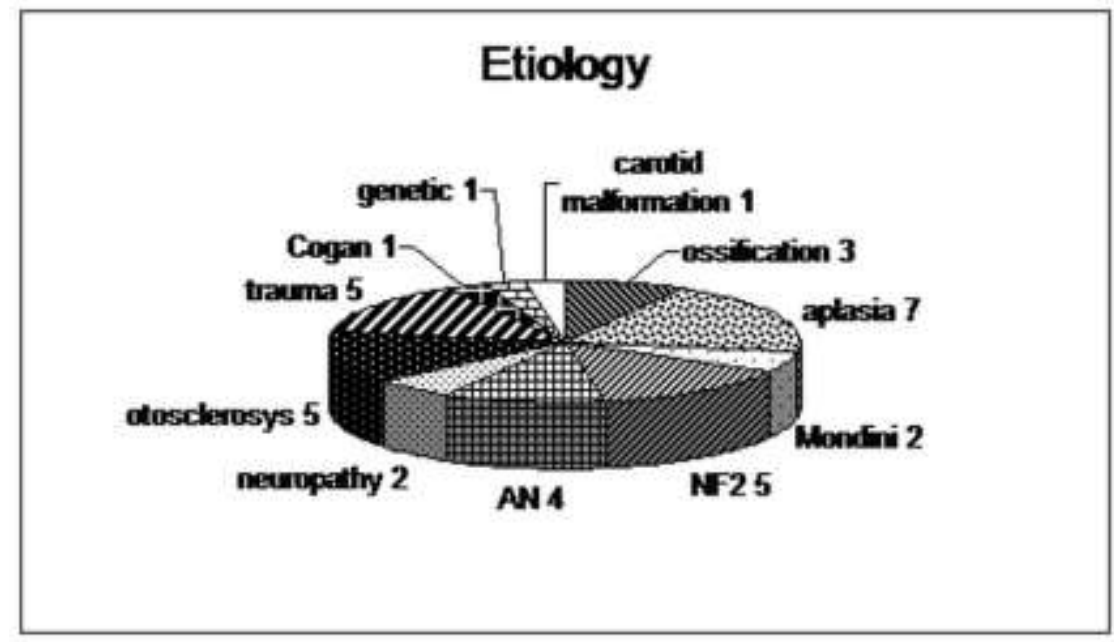

Figure 1: Aetiologies of the patients' group. Of these patients, $19.44 \%$ presented cochlear nerve aplasia, 16.89\% trauma, otosclerosis and NF2, $11.11 \%$ acoustic neuroma, $8.33 \%$ ossification, 5.56\% Mondini type II syndrome and neuropathy, 2.28\% (1 patient) Cogan syndrome, carotid malformation and genetic.

The electrodiagnostic system Amplaid MK12 (Amplifon SpA, Milan, Italy) was used to record auditory evoked potentials. For EABR recording, the positive electrode was placed on the forehead, the negative electrode on the ipsilateral tragus and the ground one on the sternum. Short recording electrode wires were used to reduce electrical interference. The electrodes wires were connected directly into an electrode head-box that was connected to the main amplifier. The use of this montage is different from the montage described by Waring [6]. It was chosen because it consistently reduces stimulus artefacts (Figure 2). The recorded signal was filtered with a band pass filter ranging from 100 to $2500 \mathrm{~Hz}$. Approximately $500-1000$ responses were averaged to achieve a good signal to noise ratio.

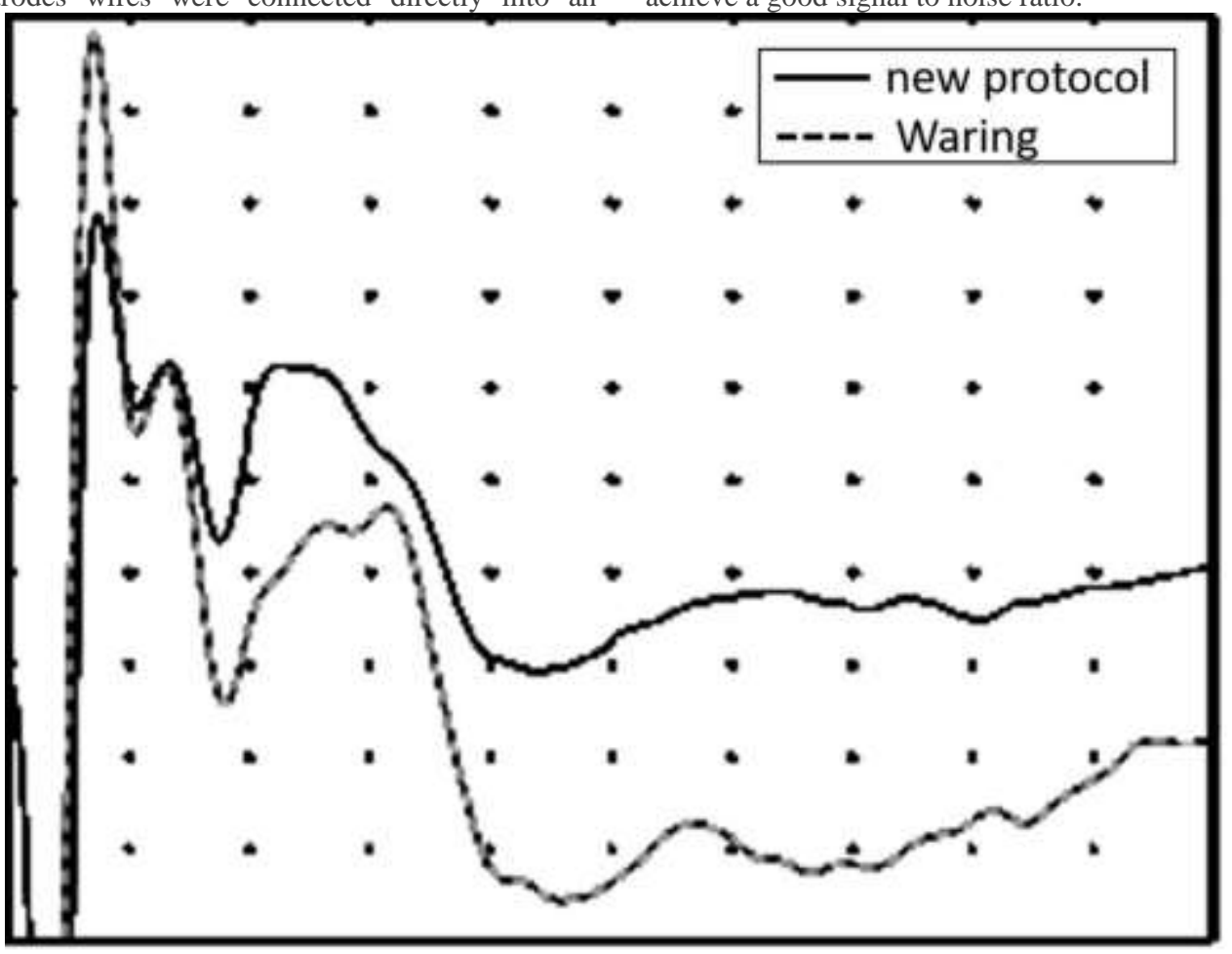

Figure 2: EABR obtained with electrode 10 as active electrode and electrode 11 as reference electrode. Waveforms are comparable. Before 1 ms, an electric artefact is presented with both recording electrodes configurations, but appears reduced in amplitude with the new combination. 
Stimulation of the ABI was executed using a Cochlear's Portable Programming System (PPS) connected to a SPrint speech processor. Stimulation was controlled via Nucleus NRT 3.1 software. The averager was triggered by directly connecting the $3.5 \mathrm{~mm}$ jack plug from the PPS to the external trigger input of the electrodiagnostic system. The speech processor was placed within a sterile sheath and the coil was positioned over the receiver antenna of the implant after $\mathrm{ABI}$ insertion.

Twenty-two patients were tested with the Cochlear suggested combination of electrodes [13], [14], fourteen with a new electrode combination (new protocol - Figure 3).

\section{(a)}

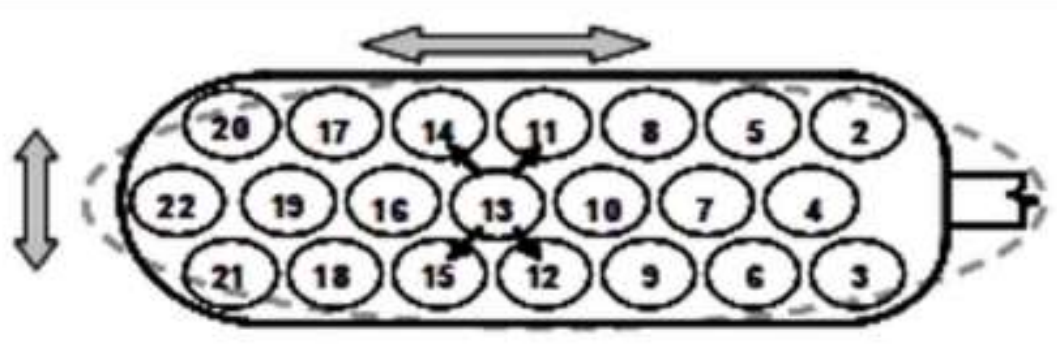

(b)

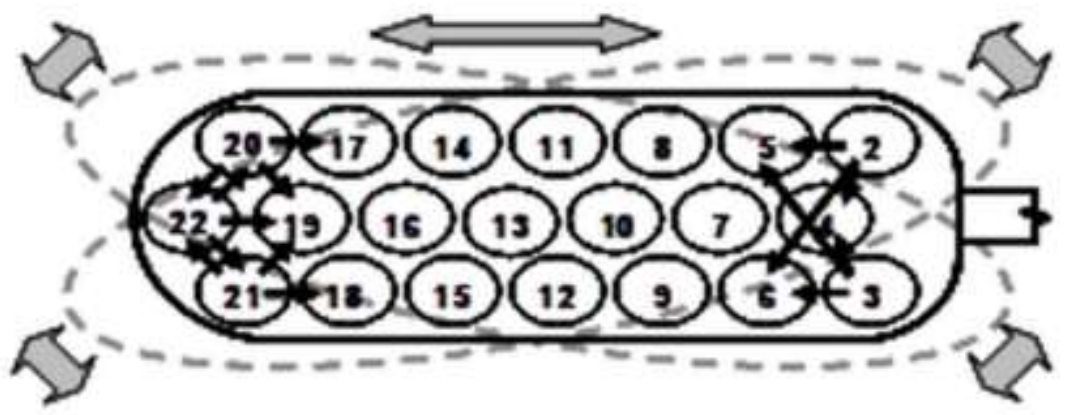

(c)

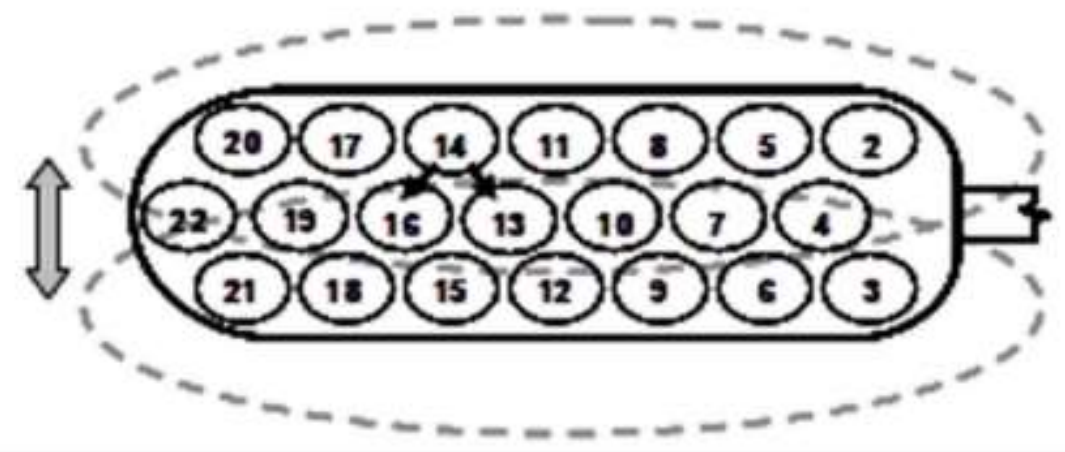

Figure 3: Electrode combinations for confirming correct electrode placement. (a) central electrode combinations to evaluate longitudinal and transversal tilt; (b) edge electrode combinations to evaluate rotational and transversal shift; (c) lateral electrode combinations to evaluate longitudinal shift.

The new protocol starts recording EABR from electrode 13 (Figure 3a), which has the highest probability to be over the cochlear nucleus surface (EABR response was obtained in $93.1 \%$ of recordings, considering electrode 13 as active electrode). Electrode 13 is stimulated with

(a)

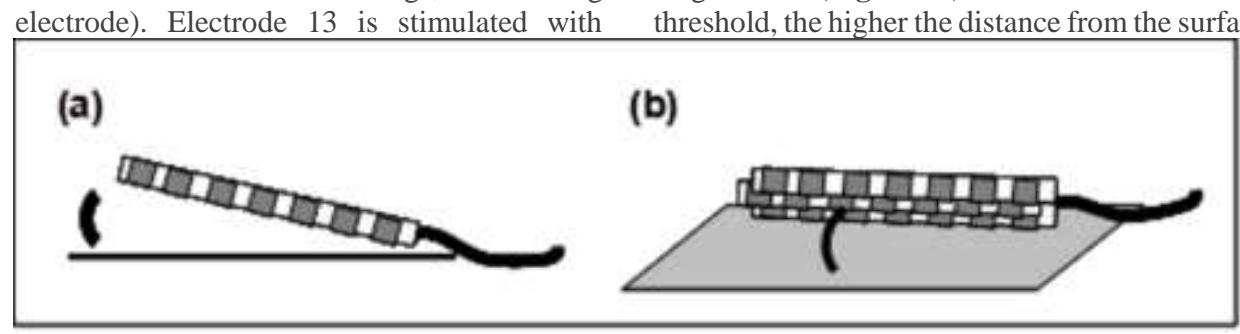

electrodes $11,12,14$ and 15 as reference electrodes to quantify longitudinal and transversal tilt of the array by evaluating peaks magnitudes (Figure 4): the smaller the amplitude, the higher the threshold, the higher the distance from the surface of the cochlear nucleus.

Figure 4: (a) Comparing EABR magnitude of electrode 13 stimulated with electrodes 11 and 14 or of electrode 13 stimulated with electrodes 12 and 15 it is possible to evaluate the longitudinal tilt of the array; (b) comparing EABR magnitude of electrode 13 stimulated with electrodes 11 and 12 or of electrode 13 stimulated with electrodes 14 and 15 it is possible to evaluate the transversal tilt of the array. 
The amplitudes analysis of EABRs recorded from the edge electrodes of the array (Figure $3 \mathrm{~b}$ ) permits to quantify both the rotational shift and the transversal axis of the array respect to the cochlear nucleus surface.

\begin{tabular}{|l|l|}
\hline Software Parameters & Settings \\
\hline Stimulation Mode & BP (change active and indifferent) \\
\hline Stimulation Modulation Type & Current Levels (CL) \\
\hline Stimulation Pulse Width & $150 \mu \mathrm{s}$ \\
\hline Stimulation Rate & $25 \mathrm{~Hz}$ \\
\hline Current Level & $190 \mathrm{CL}(469 \mu \mathrm{A}-704 \mathrm{nC})$ \\
\hline
\end{tabular}

Table 1: Settings for the software.

Software parameters are reported in Table 1. All electrode combinations were stimulated with alternate current so to cancel the stimulus artefact.
Instead, EABR recordings from the lateral electrodes of the array (Figure $3 c)$ clarifies the longitudinal axis of the array respect to the axis of the cochlear nucleus.

In order to evaluate only the acoustic response (without components of other cranial nerve stimulation, usually presented after the first $4-4.5$ $\mathrm{ms}$ ), patients were anesthetized with curare (Figure 5).

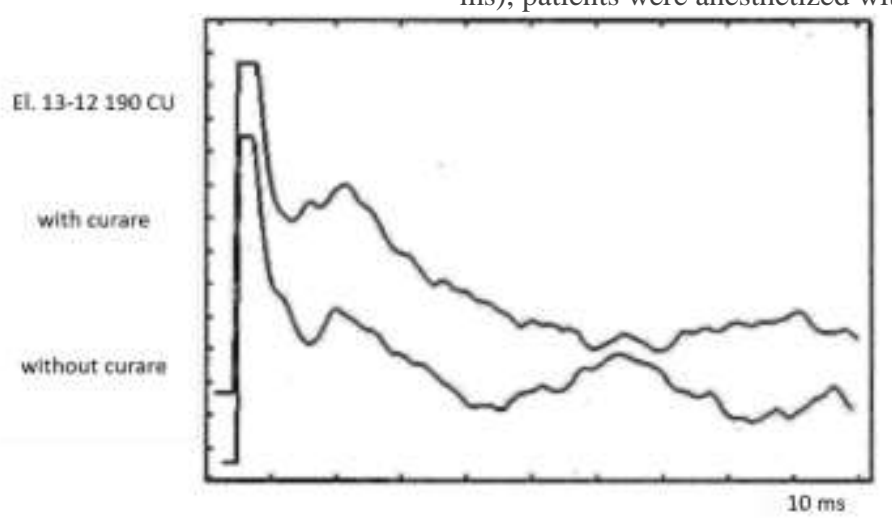

Figure 5: Effects of utilization of curare in EABR recordings. The presence of curare in trace 1 consistently reduce myogenic component at 6.4 ms.

Data were analyzed from the morphological point of view, in terms of number and amplitudes of the peaks in each recording, and considering the presence of saturation effects and of electric artefacts.

Activation occurred from 27 to 67 days from the surgery. The amount of charge needs to elicit the acoustic response was compared.

\section{Results}

In all patients, intraoperative EABR were recorded, obtaining 507 waveforms with Cochlear electrodes combination and 917 waveforms with the new procedure. Table 2 summarized the characteristics of both protocols' waveforms. Respectively, in 6.13\% (Cochlear pr.) and 6.67\% (new pr.) of cases, it was impossible to obtain an acoustic response. Most frequently a 2-peaks wave was obtained with both procedures. A significant reduction $(3.54 \%)$ of 1-peak waves was noticed with the new protocol, while there was an increase of $2.29 \%$ in 3 -peak waves presence.

\begin{tabular}{|c|c|c|}
\hline peaks & Cochlear protocol & new protocol \\
\hline 0 & $6.31 \%$ & $6.67 \%$ \\
\hline 1 & $28.60 \%$ & $25.05 \%$ \\
\hline 2 & $48.13 \%$ & $49.02 \%$ \\
\hline 3 & $16.96 \%$ & $19.26 \%$ \\
\hline
\end{tabular}

Table 2: Waveforms distinguished by number of presented peaks.

In Figure 6, peaks in each waveform are shown, considering the standard latencies of $0.7 \mathrm{~ms}(0.6 \mathrm{~ms}-1.1 \mathrm{~ms})$ for the first peak, of $1.6 \mathrm{~ms}(1.2 \mathrm{~ms}$ $-1.9 \mathrm{~ms})$ for the second peak and of $2.7 \mathrm{~ms}(2.2 \mathrm{~ms}-3.7 \mathrm{~ms})$ for the third peak [7]. Peak II was the most dominant and reliable peak for both methods. Peak I was detected $31.08 \%$ of times with the new protocol with a decrease of $6 \%$ respect to Cochlear protocol. Using the new protocol peak III was identified in $71.76 \%$ of recordings ( $10.81 \%$ more than using Cochlear protocol). 


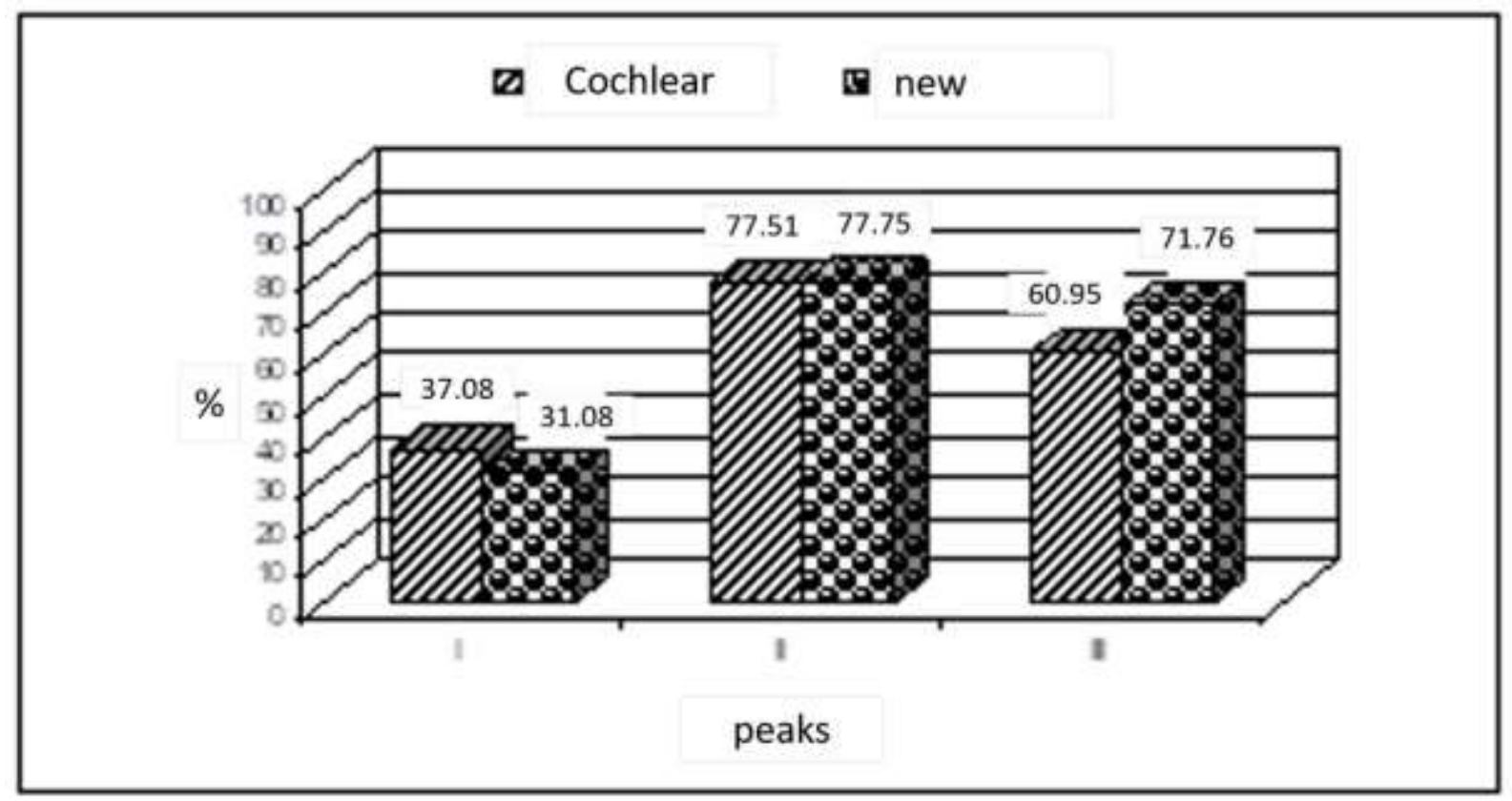

Figure 6: Peaks characterization in the recorded EABR waveforms.

Examples of EABR recordings of the same subject are shown in Figure 7. In Table 3, mean amplitude values of different peaks are presented. Magnitude of these waves is larger using Cochlear method. Particularly, this is evident for peak I. In Figure 8 is shown the EABRs recorded with an electrode combination in witch distances between the active and reference electrodes are progressively reduced. It is clear that shorter is the distance, lower is the magnitude. This means that enlarging the distance between the electrodes a larger number of fibers are elicited and so in the recorded response a sizeable component of other electrodes is presented.

\begin{tabular}{|l|c|c|c|}
\hline Peaks (nV) & I & II & III \\
\hline Cochlear protocol & 724 & 667 & 389 \\
\hline new protocol & 298 & 507 & 309 \\
\hline
\end{tabular}

TABLE 4: Mean magnitudes of EABRs peaks.

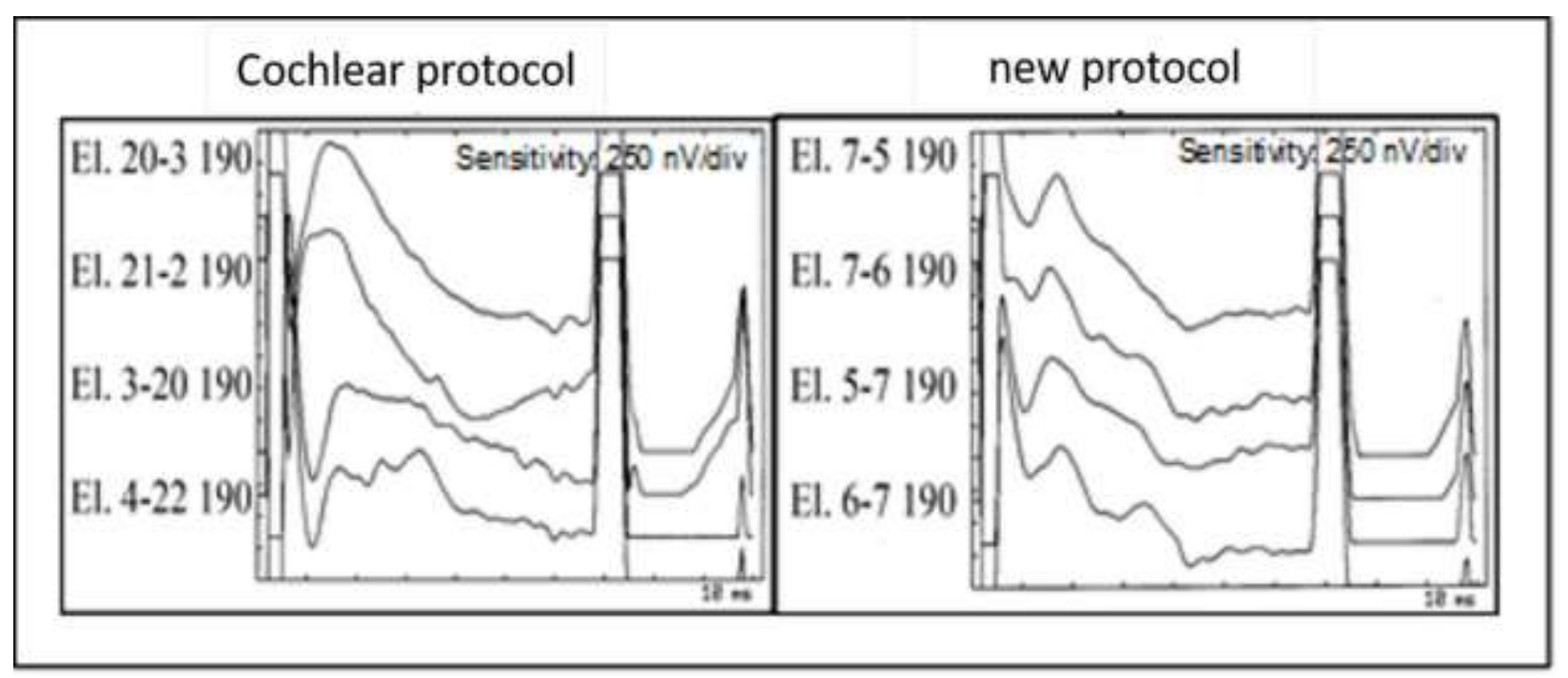




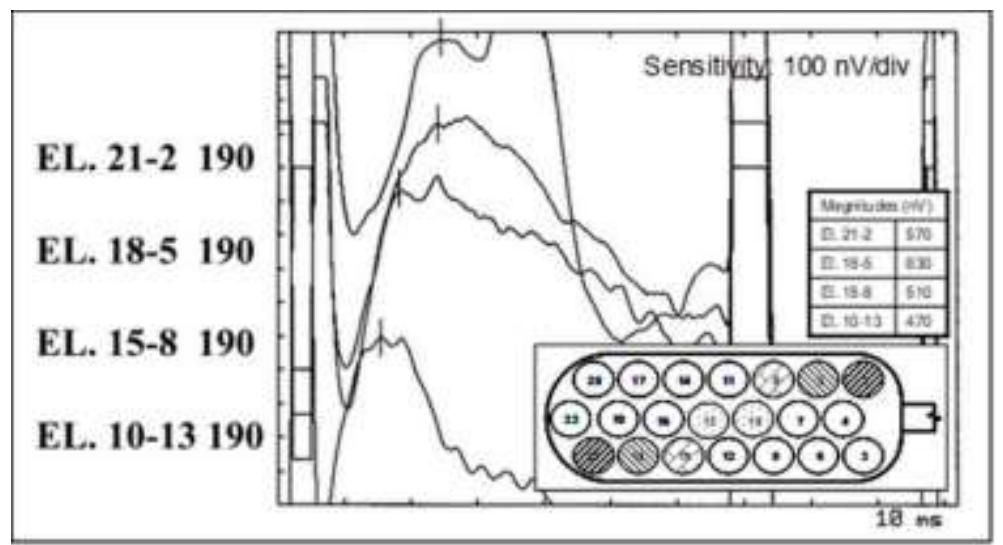

Figure 8: Effects of channel interaction on peaks magnitude: shorter is the distance between active and reference electrodes, lower is the magnitude of peaks. In this case, magnitude of EABR obtained stimulating electrode 21 with electrode 2 appears lower respect to magnitude of EABR obtained from electrode 18 stimulated with electrode 5 because electrode 21 is out of the auditory area. This leads to conclude that the recorded response is only composed by the other electrodes' contributions.

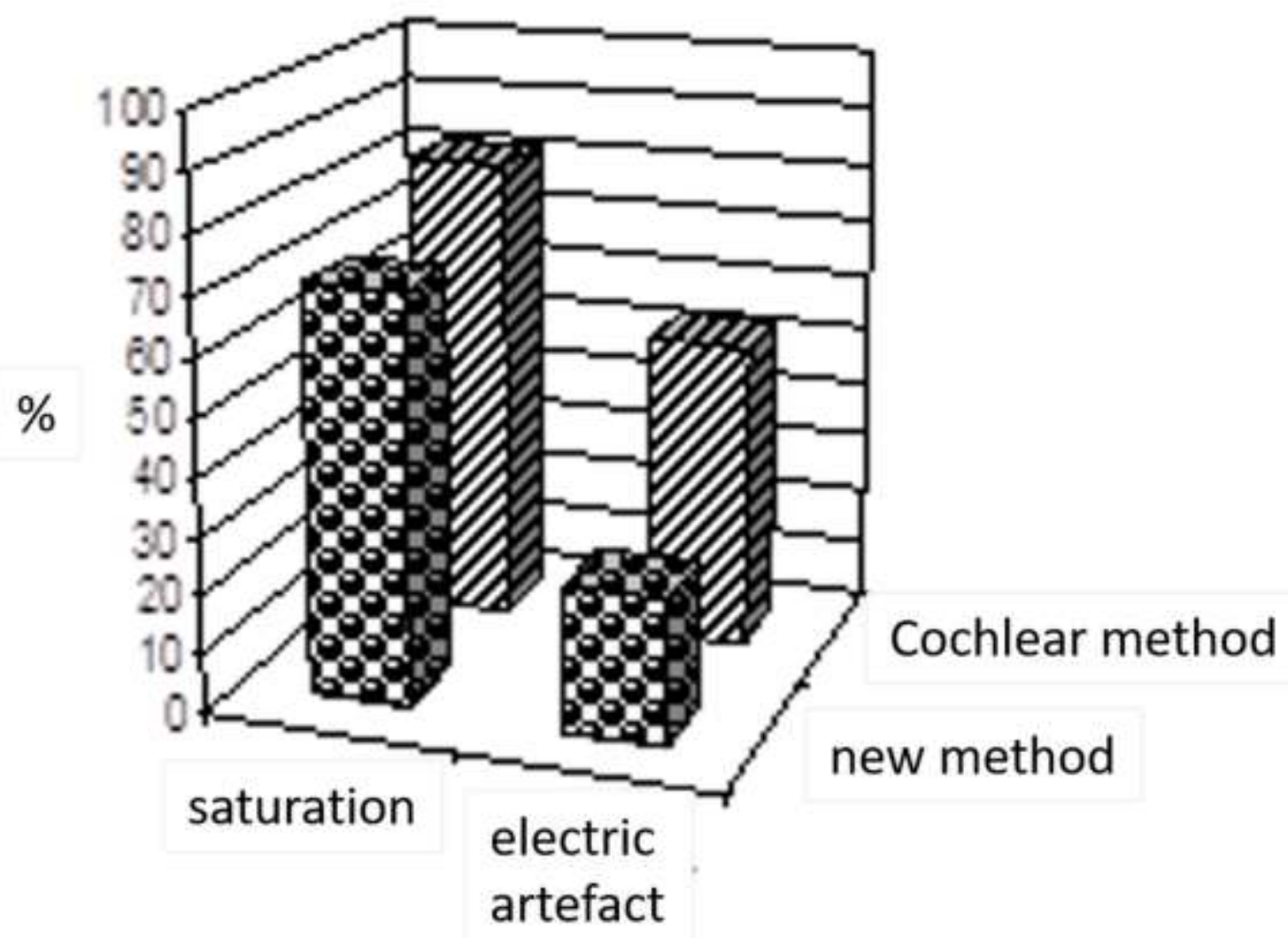

Figure 9: Percentages of saturation effects and electric artefacts present in EABR recordings in Cochlear and the new combination of electrodes protocols.

Figure 9 describes the percentage of EABRs presenting saturation effects and/or electric artefacts. Saturations effects and electric artefacts were noticed respectively in $81.85 \%$ and $53.25 \%$ of recordings using Cochlear method, while in $70.34 \%$ and $24.75 \%$ of recordings using the new method, with a percentage variation of $11.51 \%$ and $28.50 \%$.

Tuning of the implants was performed in intensive care unit. Patients were seated in a comfort position and connected to ECG monitoring equipment to supervise their heart rhythm.
At activation of $\mathrm{ABI}$, the amount of charge required resulted of $14 \mathrm{nC}$ (SD $=30, \max =137 \mathrm{nC}, \min =3 \mathrm{nC}$ ) for the patients tested with the Cochlear method, and of $8 \mathrm{nC}(\mathrm{SD}=8, \max =29 \mathrm{nC}, \min =2 \mathrm{nC})$ for the patients tested with the new method.

\section{Discussion}

In order to increase the accuracy of the evaluation of the electrodes array placement a new procedure was developed. Aim of this procedure was to reduce channel interaction, electric artefacts and saturations effects. At 
the same time, it was intended reduce the amount of electrical charge needed to activate the electrodes post-operatively.

During ABI insertion EABR were recorded with both Cochlear suggested electrodes combination and a new combination of electrodes.

Responses were obtained in the same percentage for the two methods. It is interesting the reduction of 1-peak waves noticed with the new protocol and the increase of 3-peak waves. That is, waveforms appear to be more evident.

Analyzing the different peaks, peak II was the most dominant and reliable for both methods. A significant reduction of peak I detection was observed as an increase of peak III detection. But data didn't confirm this hypothesis. On the contrary, they underlined the reductions of both these interferences. Changing perspective, it might be interested to evaluate waveforms obtained with Cochlear electrode combination. Enlarging the distance between the electrodes a larger number of fibers were elicited and so in the recorded response a sizeable component of other electrodes was presented. At this point the question was: was it possible that the first peak was due to channel interaction? Looking at the effect of channel interaction on peak magnitude (Figure 8) it can be assumed that other electrode components determined the more extensive presence of peak I in Cochlear protocol recordings.

The increase in peak III detection observed with the new procedure was explained by the saturation effect reduction.

The new procedure appears to ensure that the electrodes are stimulated with a lower current charge. And this means that the coupling obtained with the cochlear nucleus surface is better with the new procedure, being the ABI implant closer to the nucleus itself.

\section{Conclusion}

The new procedure appears to ensure that the electrodes are stimulated with a lower current charge. And this means that the coupling obtained with the cochlear nucleus surface is better with the new procedure, being the ABI implant closer to the nucleus itself.

\section{References}

1. Grayeli AB, Kalamarides M, Bouccara D, Ambert-Dahan E, Sterkers O. Auditory brainstem implant in neurofibromatosis type 2 and non-neurofibromatosis type 2 patients. Otol Neurotol. 2008;29(8):1140-1146. https://doi.org/10.1097/MAO.0b013e31818b6238.

2. Sennaroğlu L, Colletti V, Lenarz T, Manrique M, Laszig R, Rask-Andersen $\mathrm{H}$, et al. Consensus statement: Long-term results of $\mathrm{ABI}$ in children with complex inner ear malformations and decision making between $\mathrm{CI}$ and $\mathrm{ABI}$.
Cochlear Implants Int. 2016; 17(4):163-171. https://doi.org/10.1080/14670100.2016.1208396.

3. Waring M.D.: Electrical Evoked Auditory Brainstem Response Monitoring of Auditory Brainstem Integrity During Facial nerve tumor surgery. Laryngoscope, 102(11): 1293-5, 1992.

4. Brackmann D.E., Hitselberger W.E., Nelson R.A., Moore J., Waring M.D., Portillo F., Shannon R.V., Telischi F.F.: Auditory brainstem implant: I. Issues in surgical implantation. Otolaryngol. Head Neck Surg., 108(6):624-33, 1993.

5. Waring M.D.: Intraoperative electrophysiologic monitoring to assist placement of auditory brain stem implant. Ann. Otol. Rhinol. Laryngol. Suppl., 166: 33-6, 1995.

6. Waring M.D.: Properties of auditory brainstem responses evoked by intra-operative electrical stimulation of the cochlear nucleus in human subjects. Electroencephalogr. Clin. Neurophysiol., 100(6):538-48, 1996.

7. Waring M.D.: Refractory properties of auditory brain-stem responses evoked by electrical stimulation of human cochlear nucleus: evidence of neural generators. Electroencephalogr. Clin. Neurophysiol., 108(4):331-44, 1998

8. Nevison B, Laszig R, Sollmann WP, Lenarz T, Sterkers O, Ramsden R, et al. Results from a European clinical investigation of the Nucleus multichannel auditory brainstem implant. Ear Hear. 2002;23(3):170-183. https://doi.org/10.1097/00003446-200206000-00002.

9. O’Driscoll M, El-Deredy W, Ramsden RT. Brain stem responses evoked by stimulation of the mature cochlear nucleus with an auditory brain stem implant. Ear Hear. 2011;32(3):286299. https://doi.org/10.1097/AUD.0b013e3181fc9d72.

10. Wackym PA, Runge-Samuelson CL, Firszt JB. Auditory brainstem implantation. Oper Tech Otolayngol Head Neck Surg. https://doi.org/10.1016/j.otot.2005.04.004.

11. Puram SV, Herrmann B, Barker FG 2nd, Lee DJ. Retrosigmoid craniotomy for auditory brainstem implantation in adult patients with neurofibromatosis type 2. J Neurol Surg B Skull Base. 2015;76(6):440-450. https://doi.org/10.1055/s-00341544121

12. Frohne C., Matthies C., Lesinski-Schiedat A., Battmer R.D., Samii M., Lenarz T.: Extensive monitoring during auditory brainstem implant surgery. J. Laryngol. Otol. Suppl., (27):32-6, 2000 .

13. Nevison B.: A guide to the positioning of brainstem implants using intraoperative electrical auditory brainstem responses. Adv. Otorhinolaryngol., 64:154-66, 2006.

14. Surgeon's Guide for the ABI24M auditory brainstem implant. - Cochlear Ltd; Australia, 2001.

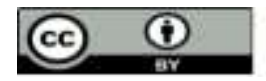

This work is licensed under Creative Commons Attribution 4.0 License
Ready to submit your research? Choose Auctores and benefit from:

$>$ fast, convenient online submission

$>$ rigorous peer review by experienced research in your field

$>$ rapid publication on acceptance

$>$ authors retain copyrights

$>$ unique DOI for all articles

$>$ immediate, unrestricted online access

At Auctores, research is always in progress.

Learn more https://auctoresonline.org/journals/neuroscience-andneurological-surgery 